\title{
Loudness judgements are not necessarily affected by visual cues to sound source distance
}

\section{Sol Libesman ${ }^{1}$, Thomas J. Whitford ${ }^{1}$, and Damien J. Mannion ${ }^{1}$}

\begin{abstract}
The level of the auditory signals at the ear depends both on the capacity of the sound source to produce acoustic energy and on the distance of the source from the listener. Loudness constancy requires that our perception of sound level, loudness, corresponds to the source level by remaining invariant to the confounding effects of distance. Here, we assessed the evidence for a potential contribution of vision, via the disambiguation of sound source distance, to loudness constancy. We presented participants with a visual environment, on a computer monitor, which contained a visible loudspeaker at a particular distance and was accompanied by the auditory delivery, via headphones, of an anechoic sound of a particular aural level. We measured the point of subjective loudness equality for sounds associated with loudspeakers at different visually-depicted distances. We report strong evidence that such loudness judgements were closely aligned with the aural level, rather than being affected by the apparent distance of the sound source conveyed visually. Similar results were obtained across variations in sound and environment characteristics. We conclude that the loudness of anechoic sounds are not necessarily affected by indications of the sound source distance as established via vision.
\end{abstract}

\section{Keywords}

audition; multisensory processing; spatial localization

\footnotetext{
${ }^{1}$ School of Psychology, UNSW Sydney

Corresponding author:

Damien J. Mannion, School of Psychology, UNSW Sydney, Australia

Email:d.mannion@unsw.edu.au
} 
The task of perception is to extract useful information about the environment from the signals available to the sensory receptors. Achieving this task often requires abstraction across the sensory effects of environmental features that are not task-relevant. For example, perceiving the physical size of an object based on visual signals requires consideration of the effect of object distance on the extent of retinal stimulation. The stable perception of environmental features, such as object size, across non task-relevant effects is termed perceptual constancy.

Here, we investigate constancy in the perception of the capacity of a sound source to produce acoustic energy - the perception of its 'loudness'. The source level of an emitting object is a feature of the environment that can aid in fundamental processes such as recognition and identification (Bizley \& Cohen, 2013). However, because sensory signals are affected by other properties of the environment, the level of a sound source cannot be established solely from examination of its direct effects on sensory receptors.

The challenge of loudness constancy can be illustrated by considering the generative process that produces auditory signals at the ear. Figure 1 depicts the key relationships under current consideration (via an influence graph, after Kersten, Mamassian, \& Yuille, 2004). The sound source has two key properties of current interest-its level (its capacity to produce acoustic energy) and its distance (its location in the environment, relative to the perceiver). Importantly, both of these properties combine to affect the auditory signals received at the ear of the perceiver. This combination means that accurate estimates of the level of the source cannot be obtained solely by evaluating the level of the aural signals received directly from the sound source, because such aural signals could be produced by any pairing of source level and distance (Bronkhorst \& Houtgast, 1999; Coleman, 1962)—with the outcome that, as stated by Worden (1971, p. 22), "intensity level, per se, holds little biological significance". For example, a given aural level could be produced by a sound source with a high level at a far distance or a sound source with a low level at a close distance.

The contribution of source level to auditory signals can be disambiguated if the distance of the sound source is identified. A potential way in which such a distance can be estimated is via the prevailing sound field, which interacts with the sound produced by the source to affect the signals at the ear (as shown in Figure 1). This sound field encompasses aspects of the environment such as the geometry and acoustic properties of surfaces and media, which interact with the emitted sound waves and affect the received auditory signals. Importantly, such interaction can produce cues to the sound source distance in the auditory signals, such as the ratio of direct to reverberant energy (see Zahorik, Brungart, \& Bronkhorst, 2005 and Kolarik, Moore, Zahorik, Cirstea, \& Pardhan, 2016, for reviews of auditory cues to distance). This estimate of sound source distance, combined with knowledge of how sound source distance and level interact in producing the auditory signals, would allow the sound source level to be disambiguated and become independent of distance. 


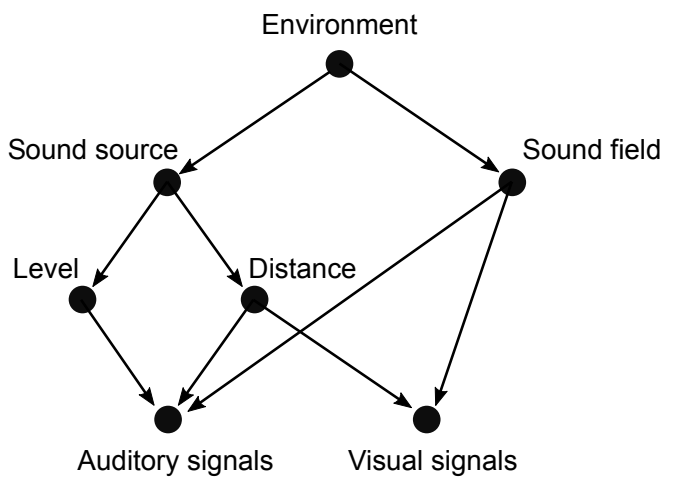

Figure 1. Influence graph for an example scenario. Nodes (circles) represent variables, and directed arrows represent causal relationships (after Kersten et al., 2004).

However, Zahorik and Wightman (2001) found that loudness constancy was evident in situations where accurate source distance judgements were not obtained. Zahorik and Wightman (2001) proposed that loudness judgements can instead be based on the energy of the reverberant component of the auditory signals, which can remain largely invariant to sound source distance in reverberant sound fields. Supporting this proposal, Altmann et al. (2013) found that loudness constancy was only present when there were strong, but not weak, reverberation cues-whereas estimates of sound source distance were not reliably affected by changes in room characteristics.

These results suggest that the capacity for loudness constancy requires the prevailing sound field to support the production of appreciable amounts of reverberant energy. However, Zahorik and Wightman (2001) also acknowledged that estimates of sound source distance obtained from visual signals may also affect loudness judgements. As shown in Figure 1, both the sound source distance and the sound field affect the visual signals. Such visual information tends to support a more accurate estimate of distance than auditory cues (Anderson \& Zahorik, 2014; Loomis, Klatzky, Philbeck, \& Golledge, 1998; Kolarik et al., 2016) and provides a comprehensive understanding of the surface structure of the environment. It would seem desirable for the mechanisms of loudness constancy to be capable of incorporating visuallydetermined distance estimates in such situations_-indeed, Calcagno, Abreg, Egua, and Vergara (2012) have proposed a key role for vision in establishing the distance of sound sources to aid in the interpretation of aural events.

Previous studies have suggested that visual information is capable of affecting loudness judgements. Mohrmann (1939, as described in Brunswik, 1956, p. 70-72) positioned pairs of loudspeakers at different distances and required participants to use the method of adjustment to equate the loudness of sounds from each loudspeaker, with the sounds presented in alternation. Participants demonstrated 
high degrees of loudness constancy, which tended to further increase when participants were able to see the scene compared to when completing the task in darkness-suggestive of a visual contribution to the mechanisms permitting loudness constancy. Similar results were obtained by Shigenaga (1965), who concluded that "there is a close relationship between the perception of acoustic distance, the constancy of loudness and the visionary cue" (p. 331). While a similar method was used by von Fieandt (1951), the contribution of vision to their reported findings is difficult to assess as performance was similarly close to complete constancy in visual and non-visual conditions.

Each of these studies were conducted under conditions in which there were likely to be concomitant auditory cues to distance. In contrast, Mershon, Desaulniers, Kiefer, Amerson, and Mills (1981) investigated the effect of visual cues on loudness judgements in an anechoic environment. Sounds were presented from a hidden loudspeaker while a silent loudspeaker was visible at different distances. Due to the visual capture phenomenon, the silent but visible loudspeaker was perceived to be the sound source. A magnitude estimation approach was used in which participants were asked to rate the loudness of the sounds. Mershon et al. (1981) found that, in an anechoic room, such loudness ratings increased when the apparent distance of the sound source changed from $75 \mathrm{~cm}$ to $225 \mathrm{~cm}$, consistent with the operation of a loudness constancy mechanism driven by visual input. However, the loudness judgements decreased to an intermediate value when the apparent distance of the sound source was further increased to $375 \mathrm{~cm}$. Hence, while providing evidence for a visual contribution to loudness, the findings of Mershon et al. (1981) are equivocal concerning the robustness and generality of such an influence.

More recently, Altmann et al. (2012) adopted a similar approach to investigating the influence of visual cues on loudness judgements. Participants heard short bursts of bandpass noise, via earphones, that were played simultaneously with the offset of a light source at varying distances. The resulting loudness ratings showed no evidence of loudness constancy, and were instead driven by the aural sound level and unaffected by the distance of the putative sound source. While potentially inconsistent with a visual contribution to loudness, it is unclear whether participants were able to accurately perceive the distance of the light source due to the limited availability of visual distance cues. Intriguingly, Altmann et al. (2012) also performed magnetoencephalography recordings which suggested that neural processing of auditory signals may have been affected by the visual distance information, despite this lack of behavioural loudness constancy.

Here, we aimed to assess the evidence for a visual contribution to loudness judgements. We constructed the simulated environment shown in Figure 2, consisting of a single visible sound source (a loudspeaker) on a grassed open-field. By rendering the loudspeaker at one of three different positions and including multiple visual cues to scene depth, we were able to manipulate the perceived distance of the apparent sound source. Furthermore, the use of an open-field environment was designed to convey the expectation of little reverberant energy (Richards \& Wiley, 1980) to the listener. Accordingly, we delivered sounds 
with a direct component only, simulating an anechoic environment in which the sound source level and distance are entirely confounded in the auditory signals.

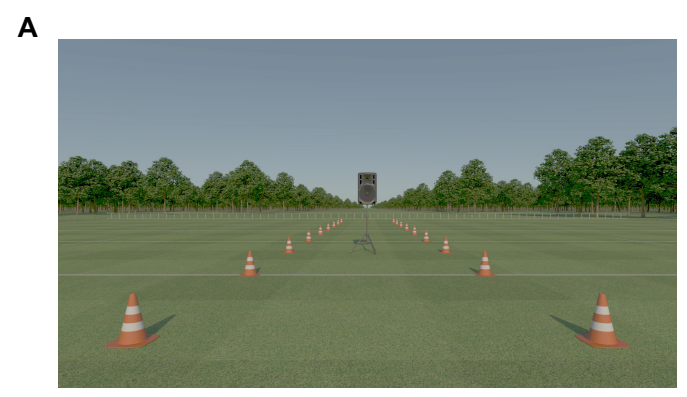

B

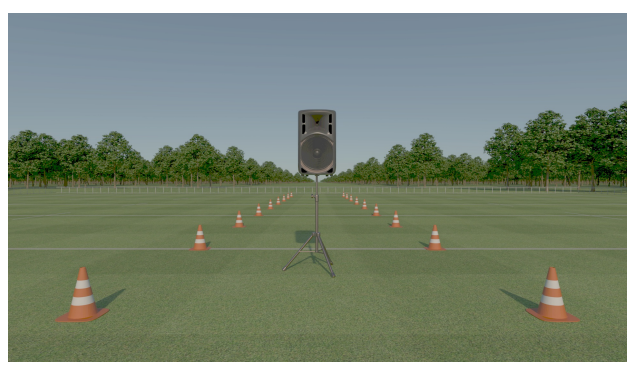

C

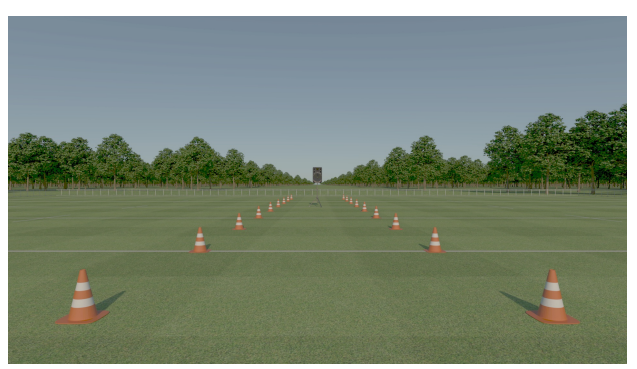

Figure 2. Visual stimuli used in Experiments 1 and 2. The loudspeaker is positioned at the reference (A), near (B), or far (C) distance from the observer.

We measured the potential influence of visual cues to sound source distance using a temporal twointerval forced-choice behavioural task, in which a reference stimulus of standard aural level and visual distance was presented in temporal proximity to a comparison stimulus of variable aural level and different visual distance (nearer or farther). By presenting the comparison with different aural levels, we identified the aural level that was required for the comparison to be perceived as equally loud as the reference. If visual signals to sound source distance can affect loudness judgements, such points of subjective loudness equality (PSEs) would have lower and higher aural levels for farther and closer 
sound sources, respectively. Alternatively, if visual signals to sound source distance are not incorporated in loudness judgements, such PSEs would be equal for farther and closer sound sources.

We performed a series of six experiments, across which we altered the auditory characteristics, the visual presentation, and the relationship between the auditory and visual presentations. In Experiments 1 and 2, participants judged the relative loudness of pure tones delivered with accompanying visual depictions of loudspeakers at different distances. In Experiment 3, this visual environment was altered to include additional visual cues to distance and to provide a visual cue that was synchronous with the auditory presentation. For Experiments 4 and 5, the auditory stimulus was changed to a pink noise burst and a speech utterance ('ba'), respectively. The final experiment introduced a distance-dependent delay between the visual onset cue and the delivery of the auditory stimulus. Overall, we find considerable evidence for the absence of a visual contribution to loudness judgements in these scenarios.

\section{Experiment 1}

\section{Method}

Participants Participants $(N=15)$ with normal or corrected-to-normal vision and no auditory pathologies (self-reported) were recruited from a pool of students enrolled in an introductory psychology course at UNSW Sydney. Participants received course credit for their involvement and gave informed and written consent in accordance with the experiment protocols approved by the Human Research Ethics Advisory Panel in the School of Psychology, UNSW Sydney (\#2683). All participants were naïve to the purposes of the experiment.

One participant was not considered due to a computer malfunction which prevented completion of the data collection session. The following analyses were conducted on the remaining 14 participants (3 males, 11 females; ages ranged from 18 to 25 with a median of 18.5).

Apparatus Auditory stimuli were presented via an 'AudioFile' device (Cambridge Research Systems, Kent, UK) and over-ear headphones (Sennheiser, Wedemark, Germany; model HD 201). The relationship between tone amplitude and the sound level produced by the headphones was determined using a artificial ear, microphone, and analyser (Brüel \& Kjæer, Nærum, Denmark; models 4152, 4144, and 2250 , respectively). All subsequently reported sound levels are in units of dB SPL as determined by this calibration method.

Visual stimuli were presented on a Display++ LCD monitor (Cambridge Research Systems, Kent, UK) with a spatial resolution of $1920 \times 1080$ pixels, temporal resolution of $120 \mathrm{~Hz}$, and mean luminance of $60 \mathrm{~cd} / \mathrm{m}^{2}$. The relationship between the video signal and monitor luminance was linear. Participants 
viewed the monitor in a darkened room from a distance of $52 \mathrm{~cm}$, via a chin rest, for a visual angular subtense of $76.6^{\circ} \times 43.1^{\circ}$.

The experiment was controlled using PsychoPy 1.83.04 (Peirce, 2007, 2008) and Python 2.7.11. The code and data from the study is available at https://bitbucket.org/account/user/ mannionlab/projects/LOUD.

Stimuli Auditory stimuli were pure tones with a frequency of $250 \mathrm{~Hz}$ and duration of 200ms, with a $5 \mathrm{~ms}$ Hanning window applied at the start and end of the waveform. Waveforms had a sampling rate of $44.1 \mathrm{kHz}$ and were identical in the left and right channels. A library of such tones was created that ranged from 50 to $80 \mathrm{~dB}$ in steps of $0.5 \mathrm{~dB}$.

Visual stimuli were depictions of an outdoor environment in which the observer was positioned on a field with a loudspeaker visible in the scene. As shown in Figure 2, the loudspeaker was positioned at a distance of $15 \mathrm{~m}$ (middle / reference), $7.5 \mathrm{~m}$ (near), or $30 \mathrm{~m}$ (far). A depiction was also produced in which the loudspeaker was not present in the scene. Images were rendered using Mitsuba (0.5.0; http://www.mitsuba-renderer.org).

Design and Procedure The experiment had a two-way within-subjects design, with factors of reference level $(66,68,70 \mathrm{~dB})$ and comparison distance (near, far). The procedure for a given participant was conducted in a single session lasting approximately one hour. The session consisted of a series of six runs, where each run assessed two combinations of reference level and comparison distance. The combinations were arranged such that they had different reference levels and comparison distances. The ordering of runs was randomised for each participant, and there was a self-paced break of at least 30 seconds between each run.

Each run consisted of a series of trials, where each trial consisted of a temporal two-interval forcedchoice task. Each interval began with a $750 \mathrm{~ms}$ preparatory period in which the scene was presented without a visible loudspeaker. The rendering with the loudspeaker at the required position was then presented for $1 \mathrm{~s}$, with the opacity increasing linearly to complete visibility over the first $75 \mathrm{~ms}$. The auditory stimulus was then delivered while the rendering remained visible for the following $700 \mathrm{~ms}$, with the opacity decreasing linearly to complete transparency over the last $75 \mathrm{~ms}$. This procedure was then repeated for the second interval (see Supplementary Video 1 for an example trial sequence). On each trial, one of the intervals contained the loudspeaker at the reference distance and the tone at the reference level while the other interval contained the loudspeaker at the comparison distance and the tone at the comparison level. The interval containing the reference was randomised on each trial. Following presentation of the two intervals, the scene was presented (with no visible loudspeaker) with a written prompt "Which interval contained the louder sound? Press the left arrow key for the first interval. Press 
the right arrow key for the second interval". The next trial commenced subsequent to the participant's button press, with a minimum inter-trial interval of 3 seconds.

The level of the comparison tone on each trial was determined using a Psi adaptive staircase procedure (Kontsevich \& Tyler, 1999). Each run contained two separate staircases, one for each different combination of reference level and comparison distance. Each consisted of 30 trials, and the staircase order was randomised within each run. As part of the staircase procedure, participant responses were modelled via a logistic-based psychometric function, after Kingdom and Prins (2010):

$$
\psi(x ; \alpha, \beta, \gamma, \lambda)=\gamma+(1-\gamma-\lambda)\left(\frac{1}{1+e^{-\beta(x-\alpha)}}\right)
$$

This psychometric function describes the probability of selecting the comparison interval as containing the louder sound for a given comparison level $(x)$, where $\gamma$ and $\lambda$ are the 'guess' and 'lapse' rates (both fixed at 0.05), $\alpha$ is the point of subjective equality (PSE), and $\beta$ is the slope. For the Psi procedure, the candidate comparison intensities were between 50 and $80 \mathrm{~dB}$ in $0.5 \mathrm{~dB}$ increments. This distribution was also used for the point of subjective equality $(\alpha)$, while the slope $(\beta)$ was given by 50 logarithmicallyspaced values between 0.1 and 10.0 .

Before commencing the experiment, the participant's dominant eye was determined using the 'card test' (described by Ehrenstein, Arnold-Schulz-Gahmen, \& Jaschinski, 2005). This was used to adjust the location of an occluder attached to the chinrest such that the participant viewed the monitor through their dominant eye only. This monocular viewing was designed to remove the influence of stereopsis cues to the true depth structure of the testing booth and promote immersion in the depicted scene.

Participants were then introduced to the task via a set of computer-based instructions (see Supplementary Table 1 for further details). They then completed a practice run, which was identical to a given random run in the experiment. Following completion of the practice run, the experimenter visually evaluated the resulting psychometric functions to determine if it appeared that participants understood the task requirements. This judgement was based on the concordance between the observed response probabilities and the assumed psychometric function-no consideration was given to the relevance to the experiment hypotheses. An additional practice run was completed if necessary.

Analysis The experiment procedure produced 360 data points per participant, where each data point specified the level of the comparison sound and the corresponding participant judgement regarding its loudness relative to the reference sound. With six conditions, this corresponded to 60 data points per condition ( 2 staircases $\times 30$ trials per staircase) per participant.

Our analysis goal was to compare the evidence for a visual contribution to loudness judgements against a null hypothesis in which loudness judgements are unaffected by concurrent visual signals. To begin, we 
define an index of vision's influence on loudness as the change in the point of subjective loudness equality with a halving of sound source distance. This index $\left(L_{v}\right)$ is in units of $\mathrm{dB}$. Under the null hypothesis, the loudness judgements are unaffected by the depiction of the sound source, to give an $L_{v}$ of zero. Under the alternative hypothesis, the depiction of the sound source affects loudness judgements such that a depiction of a nearer sound source requires a higher sound level at the ear to be perceived as equally loud as a farther sound source. For example, a point sound source in a free field with a given source level that produces $70 \mathrm{~dB}$ at the ear from the reference distance $(15 \mathrm{~m})$ would produce $76 \mathrm{~dB}$ at the ear when at the near distance $(7.5 \mathrm{~m})$ with the same source level, for an $L_{v}$ of $6 \mathrm{~dB}$.

We used a hierarchical Bayesian approach for our data analysis and hypothesis evaluation. We begin by assuming that $L_{V}$ for each participant is drawn from a normal distribution with a particular mean $\left(L_{V}^{\mu}\right)$ and standard deviation $\left(L_{V}^{\sigma}\right)$. The $L_{V}^{\mu}$ parameter is critical for our hypothesis comparison, and requires the setting of a prior that reflects our belief of the plausibility of obtaining particular values under the alternative hypothesis. As discussed above, the maximum plausible value is around $6 \mathrm{~dB}$ - however, we consider this to be relatively unlikely as the environment does not depict a point sound source or a free field, and because humans do not typically achieve complete constancy in experiment scenarios. Hence, we set the prior for $L_{V}^{\mu}$ as a normal distribution with a mean of $3 \mathrm{~dB}$ and standard deviation of 1.5 (see Figure 4 for a depiction). Setting the standard deviation to half of the mean follows the recommendation of Dienes (2014), and expresses our belief that $L_{V}^{\mu}$, under the alternative hypothesis, would be somewhere between $0 \mathrm{~dB}$ and $6 \mathrm{~dB}$, with intermediate values being more plausible. We set the prior for $L_{V}^{\sigma}$ to be vague, as a uniform distribution with lower and upper bounds of 0.01 and 3 , respectively.

With such $L_{V}$ estimates, each participant's PSEs for each of the six within-subjects conditions (two visual distances, three reference levels) can then be determined by the addition (PSE for the near conditions) and subtraction (PSE for the far conditions) of their $L_{V}$ and the reference levels. We then assume that each participant's psychometric functions have a common slope across the within-subjects conditions, and that such slopes have a prior distribution that is uniform with lower and upper bounds of $\log (0.005)$ and $\log (10)$, respectively. Finally, we model each trial in the observed data as a Bernoulli distribution, with the probability parameter given by a logistic psychometric function in Equation 1 . The alpha parameter of this logistic function is given by the estimated PSE for this participant, comparison distance, and reference level, the beta parameter is given by the estimated slope for this participant, and the catch and lapse rates are fixed at 0.05 .

The analysis model was implemented (see bayes.py in the code repository referenced in the Apparatus section) in PyMC3 (Salvatier, Wiecki, \& Fonnesbeck, 2016). Markov chain MonteCarlo (MCMC) sampling was performed using a No-U-turn sampler (Hoffman \& Gelman, 2014) with PyMC3's default initialization. A total of 20000 draws were used for each of 3 independent chains in the sampling process, after discarding the initial draws (2000) used in initializing the sampler, which were 


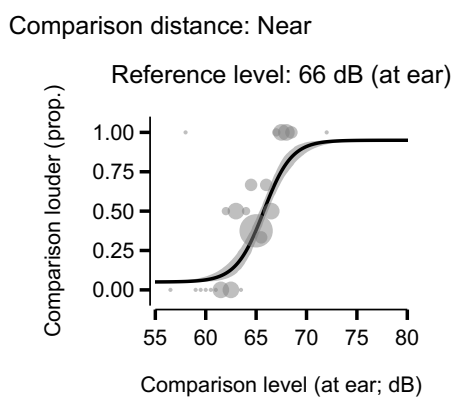

Comparison distance: Far

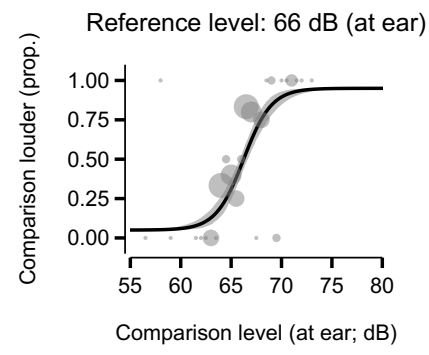

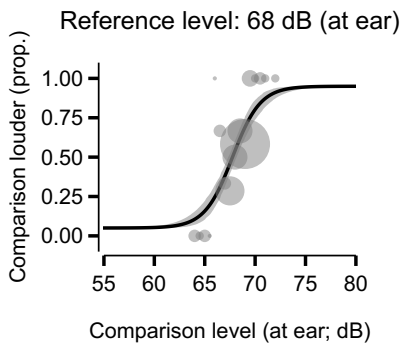

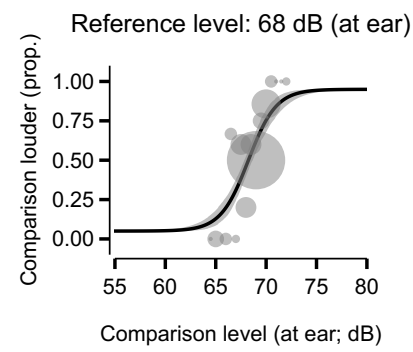

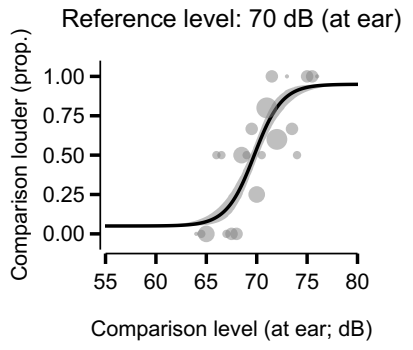

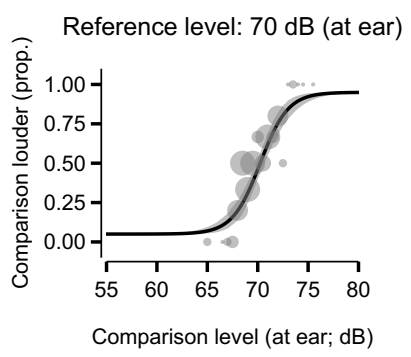

Figure 3. Psychometric functions from a representative participant in Experiment 1. Each panel depicts a single condition, with rows varying in the distance of the depicted comparison loudspeaker (near, far) and columns varying in the level at the ear of the reference sound $(66,68,70 \mathrm{~dB})$. Within each panel, points show the proportion of trials in which the participant identified the comparison sound as louder for a given comparison sound level. The point sizes are proportional to the number of trials at that particular comparison sound level. The solid line represents the mean of the posterior psychometric function distribution, and the surrounding grey area represents its 95\% HPD interval.

then concatenated and thinned by a factor of 5 to produce the posterior distributions. Sampling quality was assessed by visual inspection of sampling traces and autocorrelations, and by consideration of the match between the fitted psychometric functions and each participant's raw data (shown in Figure 3 for a representative participant and Supplementary Figure 1 for all participants).

Following model estimation, we used the Savage-Dickey method (Wagenmakers, Lodewyckx, Kuriyal, \& Grasman, 2010) to compute a Bayes factor to quantify the evidence in the comparison of the null hypothesis $\left(L_{V}^{\mu}\right.$ is $\left.0 \mathrm{~dB}\right)$ and the alternative hypothesis $\left(L_{V}^{\mu}\right.$ is most likely to be between $0 \mathrm{~dB}$ and $6 \mathrm{~dB}$, with intermediate values being more plausible). Specifically, we apply kernel density estimation (Gaussian kernel, automatic bandwidth selection using Scott's method as implemented in scipy) to the samples from $L_{V}^{\mu}$ to obtain the posterior probability at $0 \mathrm{~dB}$. The Bayes factor was then 
computed as the ratio of this posterior probability and the prior probability evaluated at $0 \mathrm{~dB}$, and is communicated as the $\log _{10}$ of this ratio.

\section{Results and Discussion}

We measured the sound level that was required for a tone emitted by a visible loudspeaker to be perceived as equally loud as a reference tone sound level emitted from a visible loudspeaker that was at a closer or farther distance. If distance estimates obtained from vision can affect loudness judgements, participants would be affected by the apparent distance of the sound emitter such that closer and farther loudspeakers would require higher and lower sound levels, respectively, to be perceived as equally loud as the reference. We constructed a hierarchical Bayesian model to compare the evidence for this hypothesis against a null hypothesis in which visually-depicted distance has no effect on loudness judgements.

We find that the posterior distribution for the mean visual influence on loudness index was close to zero, with a mean of $-0.32 \mathrm{~dB}(95 \%$ HPD $[-0.73,0.08])$. Summaries of the posterior distribution for other parameters are tabulated in Supplementary Table 2. As shown in Figure 4, the posterior density increased its mass at zero between the prior for the alternative hypothesis and the posterior. Quantification as a Bayes factor indicated moderately strong evidence for the null hypothesis against our particular alternative hypothesis $\left(\log _{10} B F_{1,0}=-1.14\right)$.

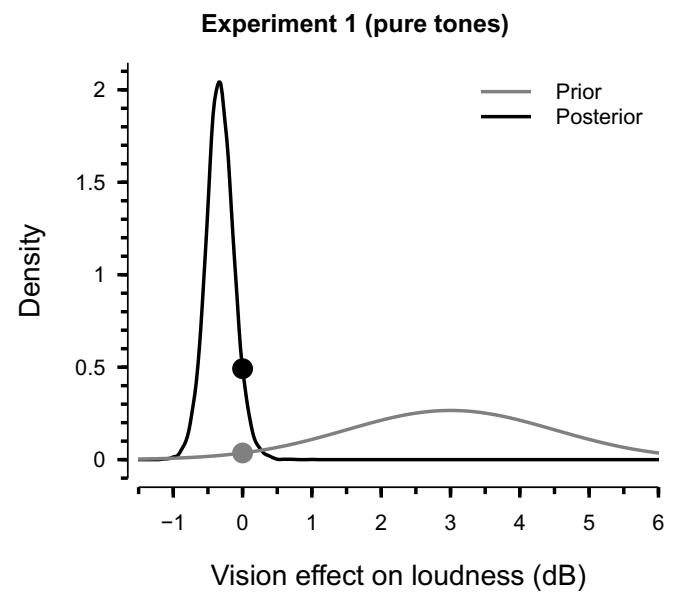

Figure 4. Evaluation of the vision effect on loudness in Experiment 1. The grey line shows the prior density for the mean vision effect on loudness $\left(L_{V}^{\mu}\right)$ parameter under the alternative hypothesis. The black line shows the posterior density for this parameter given the data obtained in Experiment 1 . The filled circles mark the density of the prior (grey circle) and posterior (black circle) at a vision effect on loudness of $0 \mathrm{~dB}$. 
The results of this experiment do not support the prediction that visual cues to distance would affect judgements of loudness in a way that would be expected from the operation of a loudness constancy mechanism. Instead, the results of this experiment provide evidence for participant judgements of loudness being unaffected by the visual signals.

Given this lack of support for the loudness constancy hypothesis in this experiment, we next conducted an experiment to probe the generality of these results. We made two primary changes in Experiment 2. First, we removed the intermediate level $(68 \mathrm{~dB})$ of the reference level factor and replaced it with a new level of the comparison distance factor in which the comparison stimulus was presented at the same distance as the reference. The rationale for this change was to encourage participants to register the differential positioning of the loudspeakers in the near and far conditions via exposure to situations in which the position of the loudspeaker does not change. Second, we changed the task instructions in an attempt to avoid biasing participants towards making their judgements based on the aural level. This involved alterations designed to promote consideration of each loudspeaker presentation as a separate object with potentially different capacities to produce sound.

\section{Experiment 2}

\section{Method}

Participants An additional set of unique participants $(N=18)$ was recruited as per the procedures for Experiment 1.

Apparatus The apparatus were as per Experiment 1.

Stimuli The stimuli were as per Experiment 1.

Design and Procedure The design and procedure were as per Experiment 1, with the exception that the reference levels were changed to 66 and $70 \mathrm{~dB}$ (the $68 \mathrm{~dB}$ reference was removed) and the comparison distances were changed to near, far, and reference (where 'reference' is an addition in which the comparison distance is the same as the reference distance). There were also wording changes to the instructions (see Supplementary Table 1).

Analysis The analysis was as per Experiment 1. Four participants were excluded due to an inability to obtain reasonable parameter estimates from their data (see Supplementary Figure 2). The following analyses were conducted on the remaining 14 participants ( 4 males, 10 females; ages ranged from 18 to 23 with a median of 19). 


\section{Results and Discussion}

Participants appeared to again be unaffected by the apparent distance from the simulated sound source. The posterior distribution for the mean visual influence on loudness index was close to zero, with a mean of $-0.07 \mathrm{~dB}(95 \% \mathrm{HPD}[-0.26,0.10])$. Summaries of the posterior distribution for other parameters are tabulated in Supplementary Table 3. As shown in Figure 5, the posterior density increased its mass at zero between the prior for the alternative hypothesis and the posterior. Quantification as a Bayes factor indicated very strong evidence for the null hypothesis against our particular alternative hypothesis $\left(\log _{10} B F_{1,0}=-1.92\right)$.

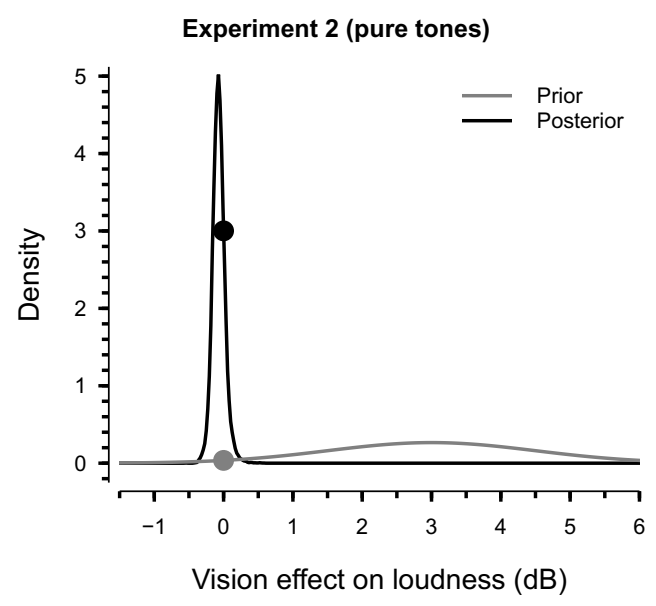

Figure 5. Evaluation of the vision effect on loudness in Experiment 2. The grey line shows the prior density for the mean vision effect on loudness $\left(L_{V}^{\mu}\right)$ parameter under the alternative hypothesis. The black line shows the posterior density for this parameter given the data obtained in Experiment 2. The filled circles mark the density of the prior (grey circle) and posterior (black circle) at a vision effect on loudness of $0 \mathrm{~dB}$.

The results of Experiment 2 are consistent with those from Experiment 1-there is no indication that participants were demonstrating loudness constancy in their judgements. Together, Experiments 1 and 2 both provided little support for the notion that visual cues to distance necessarily affect loudness judgements so as to support loudness constancy.

In Experiment 3, we sought to alter the visual stimuli to provide a more compelling impression of the environment and of the position of its visible sound sources. In particular, we were concerned that the magical appearance and disappearance of the loudspeakers in Experiments 1 and 2 may have constituted an ecological incoherence that may have prevented participants from integrating the visual cues to distance into their loudness judgements. Hence, we developed a modified environment in which the loudspeakers at each of the three distances were present simultaneously, as shown in Figure 6 (which 
is similar to the arrangement suggested by Eisler, 1981). To prevent an expectation of binaural auditory cues, we rotated the position of the camera such that the active loudspeaker was always positioned directly in front of the observer. We anticipated that the addition of such simultaneous relative size cues and rich motion parallax cues would increase the sense of immersion and of the presence of three objects at different distances.

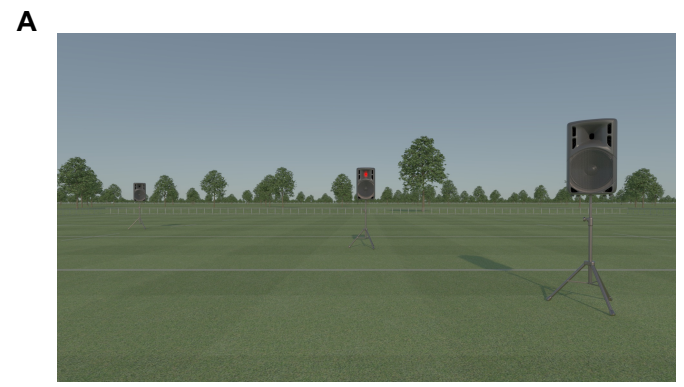

B

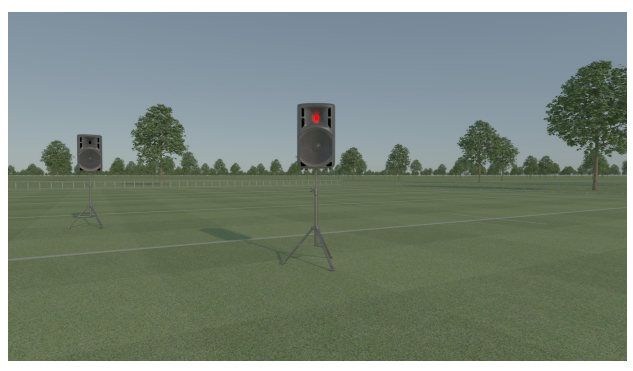

C

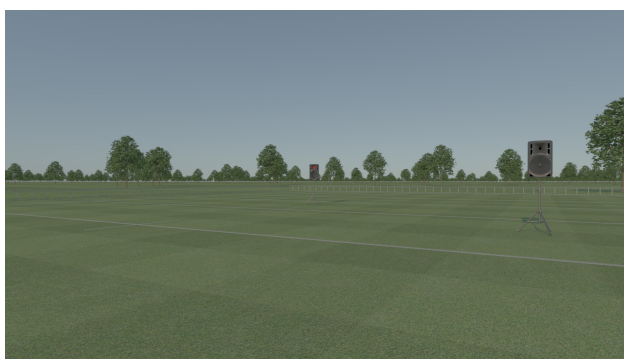

Figure 6. Sample frames from the visual stimuli used in Experiments 3-6. The camera is centred on the loudspeaker at the reference $(\mathbf{A})$, near $(\mathbf{B})$, or far $(\mathbf{C})$ distance from the observer. The renderings depict the display during presentation of the sound, which includes the presence of a red light on the central loudspeaker that is absent when the sound is not playing.

Furthermore, we introduced a visual cue to loudspeaker activation that was synchronous with the onset of the auditory stimulus. A light source at the top of each loudspeaker emitted red light while the associated sound was being played. This both served as an indication that the frontally-positioned 
loudspeaker was the source of the emitted sound and provided an audio/visual synchrony cue to aid in the perceptual binding of the sound to the loudspeaker.

\section{Experiment 3}

\section{Method}

Participants An additional set of unique participants $(N=16)$ was recruited as per the procedures for the previous experiments.

Apparatus The apparatus were as per the previous experiments.

Stimuli There were three primary changes to the visual stimuli in this experiment. First, the near and far speakers were moved horizontally to permit all three speakers (near, far, reference) to be visible simultaneously. The near and far speakers were moved such that the camera would need to rotate $30^{\circ}$ to the right and left, respectively, to focus on the speaker. Second, a light source was added to the upper section of each speaker. Additional images were rendered depicting a focus on each speaker with this light active, which emitted a red glow. Third, a set of 121 images was rendered in which the angle and distance of the camera target changed linearly between the near and far loudspeaker positions. Examples of the resulting stimuli are shown in Figure 6.

The auditory stimuli were as per the previous experiments.

Design and Procedure The presentation sequence on a given trial was changed from Experiment 2 to accommodate the dynamic transition between the camera's focus on different loudspeakers. Each interval began with a 500ms static period in which the camera was focused on the speaker from the previous interval (for the first of the two intervals, this was the second interval in the previous trial). There was then a period in which the presented image changed on each frame to display the transition to the focus loudspeaker for the interval. The duration of this period was either 500ms if the transition involved the reference distance or $1000 \mathrm{~ms}$ if it was between the near and far speakers. There was then a 750ms static period in which the camera was focused on the relevant loudspeaker, followed by the synchronous presentation of the scene with the light on the relevant loudspeaker active and the onset of the sound. The image with the active light was shown for $125 \mathrm{~ms}$, before the light was turned off and the static image displayed for an additional 500ms. This was then repeated for the second interval. An example trial sequence is shown in Supplementary Video 2.

The instructions were changed to accommodate the alterations to the visual stimuli-see Supplementary Table 1 for details. 
Analysis Two participants were excluded due to an inability to obtain reasonable parameter estimates from their data (see Supplementary Figure 3). The following analyses were conducted on the remaining 14 participants (4 males, 9 females; ages ranged from 18 to 23 with a median of 19; demographics unknown for 1 participant).

\section{Results and Discussion}

Consistent with the results of Experiments 1 and 2, participants once again appeared to be unaffected by the apparent distance from the simulated sound source. The posterior distribution for the mean visual influence on loudness index was close to zero, with a mean of $-0.16 \mathrm{~dB}$ (95\% HPD $[-0.57,0.26])$. Summaries of the posterior distribution for other parameters are tabulated in Supplementary Table 4. As shown in Figure 7, the posterior density increased its mass at zero between the prior for the alternative hypothesis and the posterior. Quantification as a Bayes factor indicated very strong evidence for the null hypothesis against our particular alternative hypothesis $\left(\log _{10} B F_{1,0}=-1.59\right)$.

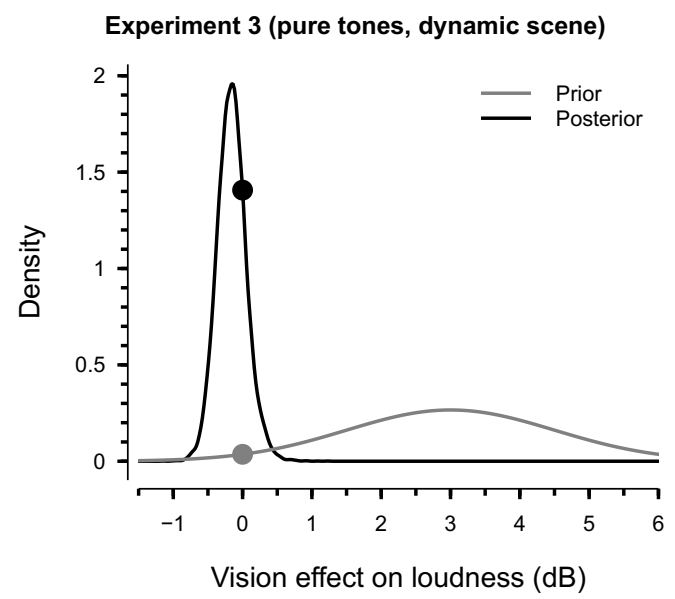

Figure 7. Evaluation of the vision effect on loudness in Experiment 3. The grey line shows the prior density for the mean vision effect on loudness $\left(L_{V}^{\mu}\right)$ parameter under the alternative hypothesis. The black line shows the posterior density for this parameter given the data obtained in Experiment 3 . The filled circles mark the density of the prior (grey circle) and posterior (black circle) at a vision effect on loudness of $0 \mathrm{~dB}$.

The alterations to the visual stimuli in this experiment had little apparent effect, with loudness judgements again being consistent with the aural level and seemingly unaffected by the apparent distance of the sound source as conveyed by visual cues. Taken together, Experiments 1-3 provide considerable evidence that loudness constancy is not necessarily achieved when cues to distance are provided solely through the visual modality. 
However, Experiments 1-3 each only considered one form of auditory stimulation-250 Hz pure tones. While an appealingly simple stimulus, pure tones may not be representative of the conditions in which loudness constancy is typically expressed. Indeed, Shigenaga (1965) reported a complex variation of the degree of loudness constancy with pure tone frequency and Mohrmann (1939, as described in Brunswik, 1956, p. 70-72) found that tones were among the forms of auditory stimulus that were least able to elicit high degrees of loudness constancy.

In the next experiment, we sought to extend the form of auditory stimulation beyond pure tones. We retained the loudspeaker depiction used in this experiment, but changed the auditory exemplars to pink noise bursts. Noise was used as the auditory stimulus in Mershon et al. (1981), who reported reported equivocal degrees of loudness constancy, and by Altmann et al. (2012), who reported a lack of behavioural loudness constancy. Mohrmann (1939, as described in Brunswik, 1956, p. 70-72) also had a condition where the stimulus was auditory noise and reported substantial degrees of loudness constancy, however such judgements were very similar regardless of the presence of visual cues.

\section{Experiment 4}

\section{Method}

Participants An additional set of unique participants $(N=15)$ was recruited as per the procedures for the previous experiments.

Apparatus The apparatus were as per the previous experiments. A calibration was performed in which the relationship between pink noise waveform root-mean-square (RMS) and the level produced by the headphones was determined and used to generate stimuli in $\mathrm{dB}$ units.

Stimuli Auditory stimuli were pink noise bursts that were $200 \mathrm{~ms}$ in duration. A library of noise samples was created that ranged from 50 to $80 \mathrm{~dB}$ in steps of $0.5 \mathrm{~dB}$. Each noise sample was created by first generating a set of phases from the frequency domain representation of a sequence drawn from a uniform distribution. These phases were then combined with amplitudes that were proportional to the inverse of the frequency between $20 \mathrm{~Hz}$ and $20 \mathrm{kHz}$ and then converted into the time domain. The resulting distribution was then $z$-scored before being multiplied by the desired RMS. A 5ms Hanning window was applied to the start and end of resulting waveform, and the same waveform was entered into the left and right stereo channels.

The visual stimuli were as per Experiment 3.

Design and Procedure The design and procedure were as per Experiment 3. See Supplementary Video 3 for a depiction of the trial sequence with the pink noise auditory stimulus. 
Analysis Two participants were excluded due to an inability to obtain reasonable parameter estimates from their data (see Supplementary Figure 4 for a depiction). The following analyses were conducted on the remaining 13 participants (6 males, 7 females; ages ranged from 18 to 21 with a median of 19).

\section{Results and Discussion}

Changing the sound from a pure tone to a pink noise burst appeared to have little influence on the results, with participants once again appearing to be unaffected by the apparent distance to the simulated sound source. The posterior distribution for the mean visual influence on loudness index was close to zero, with a mean of $0.08 \mathrm{~dB}(95 \%$ HPD $[-0.09,0.26])$. Summaries of the posterior distribution for other parameters are tabulated in Supplementary Table 5. As shown in Figure 8, the posterior density increased its mass at zero between the prior for the alternative hypothesis and the posterior. Quantification as a Bayes factor indicated very strong evidence for the null hypothesis against our particular alternative hypothesis $\left(\log _{10} B F_{1,0}=-1.90\right)$.

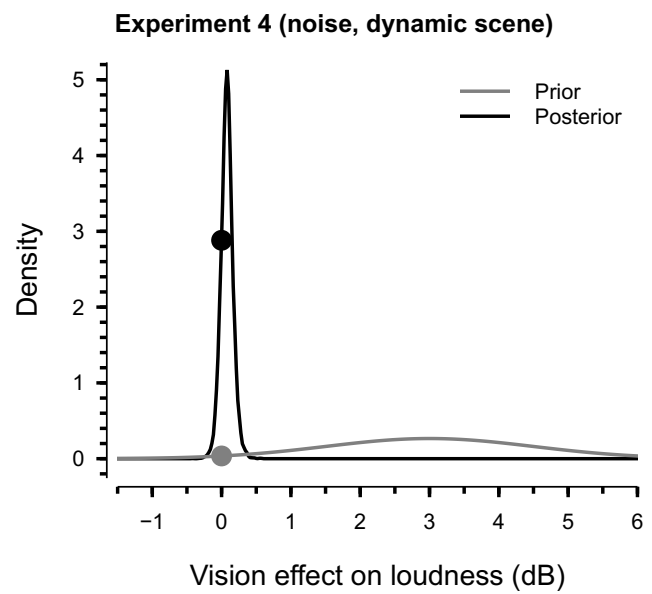

Figure 8. Evaluation of the vision effect on loudness in Experiment 4. The grey line shows the prior density for the mean vision effect on loudness $\left(L_{V}^{\mu}\right)$ parameter under the alternative hypothesis. The black line shows the posterior density for this parameter given the data obtained in Experiment 4 . The filled circles mark the density of the prior (grey circle) and posterior (black circle) at a vision effect on loudness of $0 \mathrm{~dB}$.

The aim of this experiment was to expand the consideration of the role of visual cues to sound source distance in loudness judgements beyond pure tone auditory stimuli. Hence, we replaced the presentation of pure tones with the presentation of pink noise bursts. However, the results of this experiment are very similar to those of the previous experiments using pure tones-participant judgements of loudness was 
seemingly unaffected by visual cues to the distance of the sound source and were instead closely aligned with the aural level of the noise.

In the next experiment, we expanded the range of auditory stimulation by evaluating loudness judgements to temporally-structured waveforms in which an adult male utters the syllable 'ba'. Mohrmann (1939, as described in Brunswik, 1956, p. 70-72) obtained the highest degrees of loudness constancy when speech comprised the auditory stimulation, and vocalisations produced the highest loudness constancy in the report by von Fieandt (1951). Furthermore, Rosenblum and Fowler (1991) showed that the loudness of speech can be affected by the perceived effort of the speaker as conveyed through vision.

\section{Experiment 5}

\section{Method}

Participants An additional set of unique participants $(N=23)$ was recruited as per the procedures for the previous experiments.

Apparatus The apparatus were as per the previous experiments.

Stimuli Auditory stimuli were formed from a single recording of an adult male uttering the syllable 'ba'. The waveform was approximately $275 \mathrm{~ms}$ in duration, with an $11 \mathrm{~ms}$ Hanning window applied to the beginning and end of the waveform. Intensity was manipulated by $z$-scoring the resulting waveform before multiplication by the desired RMS, and a library of samples with intensities from 50 to $80 \mathrm{~dB}$ in steps of $0.5 \mathrm{~dB}$ was formed.

The visual stimuli were as per Experiments 3 and 4.

Design and Procedure The design and procedure were as per Experiments 3 and 4. See Supplementary Video 4 for a depiction of the trial sequence with the speech utterance auditory stimulus.

Analysis Two participants were excluded due to an inability to obtain reasonable parameter estimates from their data (see Supplementary Figure 5 for a depiction), and an additional three participants were excluded due to not completing a full session. The following analyses were conducted on the remaining 18 participants (10 males, 8 females; ages ranged from 18 to 34 with a median of 19).

\section{Results}

Changing the sound from a pink noise burst to an utterance ('ba') appeared to have little influence on the results, with participants once again appearing to be unaffected by the apparent distance to the simulated 
sound source. The posterior distribution for the mean visual influence on loudness index was close to zero, with a mean of $-0.10 \mathrm{~dB}(95 \%$ HPD $[-0.20,0.00])$. Summaries of the posterior distribution for other parameters are tabulated in Supplementary Table 6. As shown in Figure 9, the posterior density increased its mass at zero between the prior for the alternative hypothesis and the posterior. Quantification as a Bayes factor indicated strong evidence for the null hypothesis against our particular alternative hypothesis $\left(\log _{10} B F_{1,0}=-1.41\right)$.

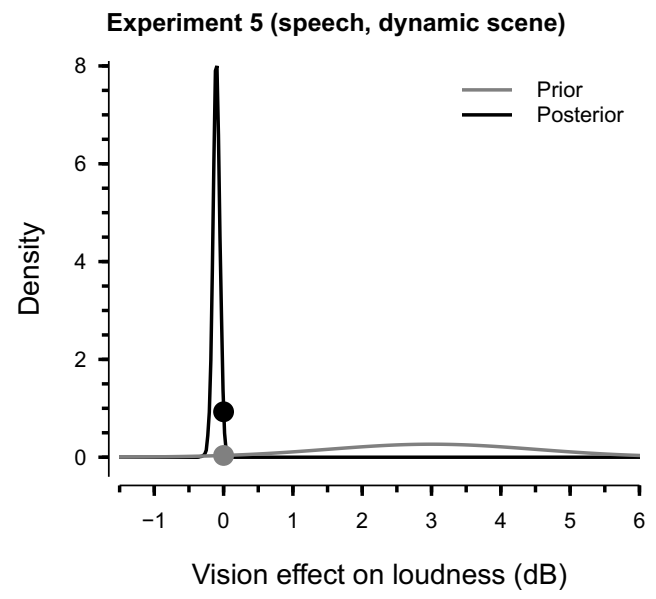

Figure 9. Evaluation of the vision effect on loudness in Experiment 5. The grey line shows the prior density for the mean vision effect on loudness $\left(L_{V}^{\mu}\right)$ parameter under the alternative hypothesis. The black line shows the posterior density for this parameter given the data obtained in Experiment 5 . The filled circles mark the density of the prior (grey circle) and posterior (black circle) at a vision effect on loudness of $0 \mathrm{~dB}$.

The aim of this experiment was to evaluate the potential role of visual cues to distance in loudness judgements for sounds mimicking human speech. As previous reports have highlighted that the loudness of speech is particularly susceptible to being affected by concomitant cues to the distance of the sound emitter (Mohrmann, 1939, as described in Brunswik, 1956, p.70-72; Pollack, 1952; von Fieandt, 1951), we expected that this would provide an opportune stimulus for demonstrating the capacity for visual cues to inform loudness judgements. However, consistent with the results of Experiments 1-4, we find that the loudness judgements were closely aligned with the aural level of the utterance and were only slightly affected by the perceived distance of the sound emitter.

In Experiments 3-5, we added a visual indicator that flashed on the currently active loudspeaker coincident with the onset of the auditory stimulus. The purpose of this indicator was to both emphasise which of the three loudspeakers was currently producing the sound and to promote the binding of the auditory and visual signals through temporal synchrony. However, this approach does not reflect the 
ecological constraint that sound travels more slowly than light—and there is evidence to suggest that the perceptual system incorporates such knowledge (Jaekl, Seidlitz, Harris, \& Tadin, 2015; Sugita \& Suzuki, 2003). Hence, the inclusion of a distance-dependent audiovisual delay may be more effective in promoting binding than audiovisual simultaneity - and may also provide a new multisensory cue to sound source distance. We evaluated this possibility in Experiment 6.

\section{Experiment 6}

\section{Method}

Participants An additional set of unique participants $(N=19)$ was recruited as per the procedures for the previous experiments.

Apparatus The apparatus were as per the previous experiments.

Stimuli The auditory stimuli were as per Experiment 5, and the visual stimuli was as per Experiments 3-5. However, the onset of the auditory stimulus was delayed relative to the onset of the 'active light' on the focused loudspeaker. The delays were approximately 22, 43, and 87 milliseconds for the near, reference, and far distances, respectively.

Design and Procedure The design and procedure were as per Experiments 3-5.

Analysis No participants were required to be excluded (see Supplementary Figure 6), and the following analyses were conducted on the complete set of 19 participants. No demographic information was collected for this experiment.

\section{Results}

The addition of a distance-dependent asynchrony between the auditory and visual indicator (light flash on the active speaker) appeared to have little influence on the results, with participants once again appearing to be unaffected by the apparent distance to the simulated sound source. The posterior distribution for the mean visual influence on loudness index was close to zero, with a mean of $-0.01 \mathrm{~dB}(95 \%$ HPD $[-0.11,0.09])$. Summaries of the posterior distribution for other parameters are tabulated in Supplementary Table 7. As shown in Figure 10, the posterior density increased its mass at zero between the prior for the alternative hypothesis and the posterior. Quantification as a Bayes factor indicated decisive evidence for the null hypothesis against our particular alternative hypothesis $\left(\log _{10} B F_{1,0}=\right.$ $-2.36)$. 


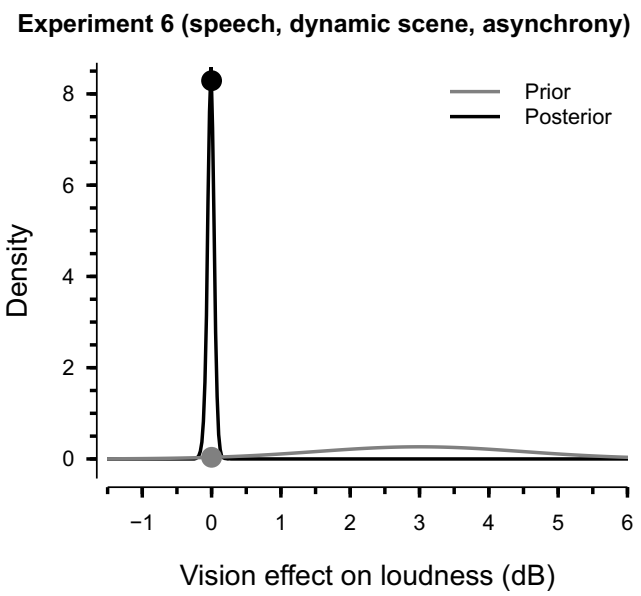

Figure 10. Evaluation of the vision effect on loudness in Experiment 6 . The grey line shows the prior density for the mean vision effect on loudness $\left(L_{V}^{\mu}\right)$ parameter under the alternative hypothesis. The black line shows the posterior density for this parameter given the data obtained in Experiment 6 . The filled circles mark the density of the prior (grey circle) and posterior (black circle) at a vision effect on loudness of $0 \mathrm{~dB}$.

The aim of this experiment was to evaluate the influence of distance-dependent audiovisual asynchrony on loudness judgements. As previous reports have suggested that the perceptual system is aware of the likely differences in the timing of auditory and visual stimulation from sound sources at a distance (Jaekl et al., 2015; Sugita \& Suzuki, 2003), we were concerned that the audiovisual synchrony in Experiments 3-5 may have reduced the perceived binding of the auditory and visual stimulation or confounded the interpretation of the apparent distance of the sound source. However, we obtained results that were consistent with those from Experiments 3-5; that is, there was little indication that the loudness judgements were affected by the apparent distance of the sound source. Although the capacity of the perceptual system to utilise audiovisual delays is controversial (Arnold, Johnston, \& Nishida, 2005; Lewald \& Guski, 2004), this result from Experiment 6 argues against the absence of meaningful audiovisual asynchrony as being the critical determinant in the apparent lack of a visual contribution to loudness judgements in this series of experiments.

\section{General Discussion}

The aim of this study was to assess the evidence for a visual contribution to loudness. By using a simulated outdoor scene with a visible loudspeaker as the sound source, we measured how human participants' judgements of relative loudness are affected by the depiction of sound source distance. In a series of six experiments, we find strong evidence that this visual information was not incorporated into 
the loudness judgements-which were instead consistent with the level of the auditory signals received at the ear. This result was obtained for pure tones, pink noise bursts, and speech utterances and across variations of the visual and audiovisual environment.

This apparent lack of a visual contribution to loudness is consistent with the behavioural findings reported by Altmann et al. (2012). Such an outcome is also potentially consistent with the anechoic experiment reported by Mershon et al. (1981) which, as described in the Introduction, was unclear in the strength of evidence for a visual contribution to loudness. Importantly, it is also in agreement with the proposal by Zahorik and Wightman (2001) that the key determinant of loudness is reverberant energy. However, this absence of a visual influence is inconsistent with studies that have reported that judgements become closer to constancy when visual information is available (Mershon, 1981; Mohrmann, 1939, as described in Brunswik, 1956, p. 70-72; Shigenaga, 1965). The key discriminating feature of such studies, relative to those reporting a lack of a visual contribution to loudness (such as the current study), appears to be that they were each conducted in reverberant rather than anechoic environments. This distinction implies that the presence of reverberation is required for vision to influence loudness.

Why might the presence of a reverberant environment be a necessary precondition for vision to inform loudness judgements? We suggest two possible explanations. First, the perceptual system may have an expectation about the likely reverberation that would be elicited when a sound source produces acoustic energy in the environment (Traer \& McDermott, 2016). The absence of such reverberation may produce a perceptual conflict that is resolved in favour of independence, rather than incorporation, of the auditory and visual information - that is, vision may not contribute to the interpretation of auditory signals because vision and audition are considered to be relating to different environmental sources. When reverberation is present, this may be sufficient to localise the sound source as being external to the perceiver and allow the system to incorporate information provided by vision. Second, reverberation may be necessary because the relevant information provided by vision pertains to reverberation. Thus far, we have focused on the potential for vision to identify the distance of the sound source. However, as mentioned in the Introduction and shown in Figure 1, vision also has the potential ability to obtain information about the prevailing sound field. For example, vision can allow the identification of the reflective surfaces in the environment-which can provide estimates of parameters relating to reverberation such as room size (Calcagno et al., 2012). This information could then be incorporated into the interpretation of auditory signals, such as by supporting the discrimination of the direct and reverberant components, thereby affecting loudness in situations with appreciable reverberant energy.

The apparent necessity of reverberation for loudness constancy has the intriguing consequence that perception would be unable to sustain constancy in anechoic or weakly reverberant environments. Indeed, a dependence of loudness on the strength of reverberation was reported by Altmann et al. (2013). The 
ecological foundations, and potential behavioural consequences, of this apparent failure of perceptual constancy is an interesting avenue for future research.

Finally, it is relevant to consider whether the task requirements in the current experiments may have obscured any loudness constancy mechanisms. Specifically, participants were instructed to judge the relative loudness of sounds emitted by objects in the environment (see Supplementary Table 1 for the wording used in each experiment); if this was interpreted as requiring to make judgements based on the signal level at the ear, we may not have captured a normal perceptual experience in which loudness constancy is evident. However, the presence of a perceptual constancy mechanism is often such that judgements are pulled in the direction of constancy even when attempting to consider sensory cues in isolation. Indeed, Mohrmann (1939, as described in Brunswik, 1956, p. 70-72) reported considerable loudness constancy with the availability of visual cues even when participants were explicitly instructed to adopt an attitude towards aural level. Hence, we suggest that task instructions are unlikely to completely explain the lack of loudness constancy that we observed in this study.

\section{Conclusion}

Despite providing information about the distance of a sound source and its environment, vision does not necessarily affect loudness judgements. Under anechoic conditions, loudness appears to be determined by the level of aural signals at the ear irrespective of concurrent visual information. This is consistent with the necessity of reverberation for loudness constancy, and the potential role of vision in such reverberant environments is an important direction for future research.

\section{Acknowledgements}

Thanks to Chris Donkin for analysis advice.

\section{Funding}

This research was supported in part by grants from the National Health and Medical Research Council of Australia (APP1090507 to T.W.) and the Australian Research Council (DP140104394 and DP170103094 to T.W.).

\section{References}

Altmann, C. F., Matsuhashi, M., Votinov, M., Goto, K., Mima, T., \& Fukuyama, H. (2012). Visual distance cues modulate neuromagnetic auditory $\mathrm{N} 1 \mathrm{~m}$ responses. Clinical Neurophysiology, 123(11), 2273-2280. 
Altmann, C. F., Ono, K., Callan, A., Matsuhashi, M., Mima, T., \& Fukuyama, H. (2013). Environmental reverberation affects processing of sound intensity in right temporal cortex. European Journal of Neuroscience, 38, 3210-3220.

Anderson, P. W., \& Zahorik, P. (2014). Auditory/visual distance estimation: accuracy and variability. Frontiers in Psychology, 5, 1097.

Arnold, D. H., Johnston, A., \& Nishida, S. (2005). Timing sight and sound. Vision Research, 45, $1275-1284$.

Bizley, J. K., \& Cohen, Y. E. (2013). The what, where and how of auditory-object perception. Nature Reviews. Neuroscience, 14, 693-707.

Bronkhorst, A. W., \& Houtgast, T. (1999). Auditory distance perception in rooms. Nature, 397, 517-520.

Brunswik, E. (1956). Perception and the representative design of psychological experiments (2nd ed.). Berkeley, CA: University of California Press.

Calcagno, E. R., Abreg, E. L., Egua, M. C., \& Vergara, R. (2012). The role of vision in auditory distance perception. Perception, 41, 175-192.

Coleman, P. D. (1962). Failure to localize the source distance of an unfamiliar sound. The Journal of the Acoustical Society of America, 34(3), 345-346.

Dienes, Z. (2014). Using Bayes to get the most out of non-significant results. Frontiers in Psychology, 5,781 .

Ehrenstein, W. H., Arnold-Schulz-Gahmen, B. E., \& Jaschinski, W. (2005). Eye preference within the context of binocular functions. Graefe's Archive for Clinical and Experimental Ophthalmology, 243, 926-932.

Eisler, H. (1981). Sensations, correlates and judgments: Why physics? Behavioural and Brain Sciences, 4, 193-194.

Hoffman, M. D., \& Gelman, A. (2014). The No-U-Turn sampler: Adaptively setting path lengths in Hamiltonian Monte Carlo. Journal of Machine Learning Research, 15, 1593-1623.

Jaekl, P., Seidlitz, J., Harris, L. R., \& Tadin, D. (2015). Audiovisual delay as a novel cue to visual distance. PloS One, 10, e0141125.

Kersten, D., Mamassian, P., \& Yuille, A. (2004). Object perception as Bayesian inference. Annual Review of Psychology, 55, 271-304.

Kingdom, F. A., \& Prins, N. (2010). Psychophysics: A practical introduction. London, UK: Academic Press.

Kolarik, A. J., Moore, B. C. J., Zahorik, P., Cirstea, S., \& Pardhan, S. (2016). Auditory distance perception in humans: a review of cues, development, neuronal bases, and effects of sensory loss. Attention, Perception, \& Psychophysics, 78(2), 373-395. 
Kontsevich, L. L., \& Tyler, C. W. (1999). Bayesian adaptive estimation of psychometric slope and threshold. Vision Research, 39(16), 2729-2737.

Lewald, J., \& Guski, R. (2004). Auditory-visual temporal integration as a function of distance: no compensation for sound-transmission time in human perception. Neuroscience Letters, 357, 119122.

Loomis, J. M., Klatzky, R. L., Philbeck, J. W., \& Golledge, R. G. (1998). Assessing auditory distance perception using perceptually directed action. Perception \& Psychophysics, 60, 966-980.

Mershon, D. H., Desaulniers, D. H., Kiefer, S. A., Amerson, T., Jr, \& Mills, J. T. (1981). Perceived loudness and visually-determined auditory distance. Perception, 10(5), 531-543.

Peirce, J. W. (2007). PsychoPy-Psychophysics software in Python. Journal of Neuroscience Methods, $162,8-13$.

Peirce, J. W. (2008). Generating stimuli for neuroscience using PsychoPy. Frontiers in Neuroinformatics, 2,10 .

Pollack, I. (1952). On the measurement of the loudness of speech. The Journal of the Acoustical Society of America, 24(3), 323-324.

Richards, D. G., \& Wiley, R. H. (1980). Reverberations and amplitude fluctuations in the propagation of sound in a forest: implications for animal communication. The American Naturalist, 115(3), 381-399.

Rosenblum, L. D., \& Fowler, C. A. (1991). Audiovisual investigation of the loudness-effort effect for speech and nonspeech events. Journal of Experimental Psychology: Human Perception and Performance, 17, 976-985.

Salvatier, J., Wiecki, T. V., \& Fonnesbeck, C. (2016). Probabilistic programming in Python using PyMC3. PeerJ Computer Science, 2, e55.

Shigenaga, S. (1965). The constancy of loudness and of acoustic distance. Bulletin Faculty Literature, Kyиshu University, 9, 289-333.

Sugita, Y., \& Suzuki, Y. (2003). Audiovisual perception: Implicit estimation of sound-arrival time. Nature, 421, 911.

Traer, J., \& McDermott, J. H. (2016). Statistics of natural reverberation enable perceptual separation of sound and space. Proceedings of the National academy of Sciences of the United States of America, 113, E7856-E7865.

von Fieandt, K. (1951). Loudness invariance in sound perception. Acta Psychologica Fennica, 1, 9-20.

Wagenmakers, E.-J., Lodewyckx, T., Kuriyal, H., \& Grasman, R. (2010). Bayesian hypothesis testing for psychologists: a tutorial on the Savage-Dickey method. Cognitive Psychology, 60(3), 158-189.

Worden, F. G. (1971). Hearing and the neural detection of acoustic patterns. Behavioral Science, 16(1), 20-30. 
Zahorik, P., Brungart, D. S., \& Bronkhorst, A. W. (2005). Auditory distance perception in humans: A summary of past and present research. Acta Acustica united with Acustica, 91(3), 1610-1928.

Zahorik, P., \& Wightman, F. L. (2001). Loudness constancy with varying sound source distance. Nature Neuroscience, 4, 78-83. 